\title{
An efficient synthesis of novel sucrose-containing dilactams
}

\author{
Mykhaylo A. Potopnyk · Sławomir Jarosz
}

Received: 27 September 2012/ Accepted: 29 November 2012/Published online: 17 January 2013

(C) The Author(s) 2013. This article is published with open access at Springerlink.com

\begin{abstract}
An efficient and convenient approach to sucrosecontaining dilactams has been developed. The method, based on reaction of regioisomeric 6,6'-di-O-[(aminomethyl)phenyl]-1',2,3,3',4,4'-hexa- $O$-methylsucrose with isophtaloyl or 2,6-pyridinedicarbonyl dichlorides, provided the 1:1-macrocycles in good yields.
\end{abstract}

Keywords Carbohydrates $\cdot$ Macrocycles $\cdot$ Alkylation · Reductions · Cyclization

\section{Introduction}

Macrocyclic compounds are important in supramolecular chemistry [1]. Especially interesting are chiral receptors capable of enantioselective complexation of a variety of important chiral guests. Carbohydrates, inexpensive, renewable raw materials available optically pure, are particularly useful in planning and executing the synthesis of such chiral hosts. The different configurations and conformations of carbohydrates can be incorporated in the target macrocycle, which makes these compounds convenient chiral synthetic analogs of poly(ethylene glycol) (PEG) reagents [2].

Chiral crown and aza-crown ethers with carbohydrate scaffolds have been extensively used as chiral catalysts in asymmetric synthesis (asymmetric epoxidation of chalcones [3-5], Michael addition [3, 4, 6, 7], and Darzens reactions $[3-5,7,8])$. Carbohydrate-containing macrocycles have also

M. A. Potopnyk $\cdot$ S. Jarosz $(\bowtie)$

Institute of Organic Chemistry, Polish Academy of Sciences, Kasprzaka 44/52, 01-224 Warsaw, Poland

e-mail: slawomir.jarosz@icho.edu.pl been investigated as fluorescent molecular sensors for cations $[9,10]$ and anions [11].

Sucrose, the most common disaccharide occurring in nature, is a promising building block for synthesis of such chiral macrocyclic receptors [12-14]. Its aza-crown derivatives enabled highly enantioselective complexation of the (S)-1-phenylethylammonium cation [15].

Isophthalic and pyridine-2,6-diamides, because of their proton-donor properties, are convenient scaffolds used as building blocks in the synthesis of macrocyclic receptors designed for complexation of anions [16], ion pairs [17], zwitterions [18], and amino acid derivatives [19]. The anion-complexing properties of such diamides have been exploited in templated syntheses of catenane [20] and rotaxane [21] systems. Macrocycles incorporating the pyridine-2,6-diamide functionality are known as molecular turnstiles [22]. Combination of the sucrose scaffold with isophthalic or pyridine-2,6-diamide units may be useful means of synthesis of a new type of chiral receptor with interesting properties.

Very recently, we reported an effective procedure for synthesis of $1^{\prime}, 2,3,3^{\prime}, 4,4^{\prime}$-hexa- $O$-methyl-6,6'-di- $O$-(methylsulfonyl)sucrose (1; four steps, $48 \%$ overall yield) which was used for preparation of macrocyclic bis-amides 3a-3c and 4a-4c (Scheme 1). Condensation of dimesylate 1 with 2 equiv. of the appropriate nitrophenol (ortho, meta, or para) followed by reduction of the nitro groups provided the expected $6,6^{\prime}$-di- $O$-(aminophenyl)-1',2,3,3',4,4'-hexa$O$-methylsucroses (2a-2c). Reaction of dianilines 2a and $\mathbf{2 b}(o$ or $m)$ with isophthaloyl or 2,6-pyridinedicarbonyl dichlorides (5 and $\mathbf{6}$ ) afforded the monomeric macrocycles in excellent yields, whereas reaction of the $p$-diamines furnished dimers as the major products (Scheme 1), with smaller amounts of the expected monomers $\mathbf{3 c}, \mathbf{4 c}$ [23]. 


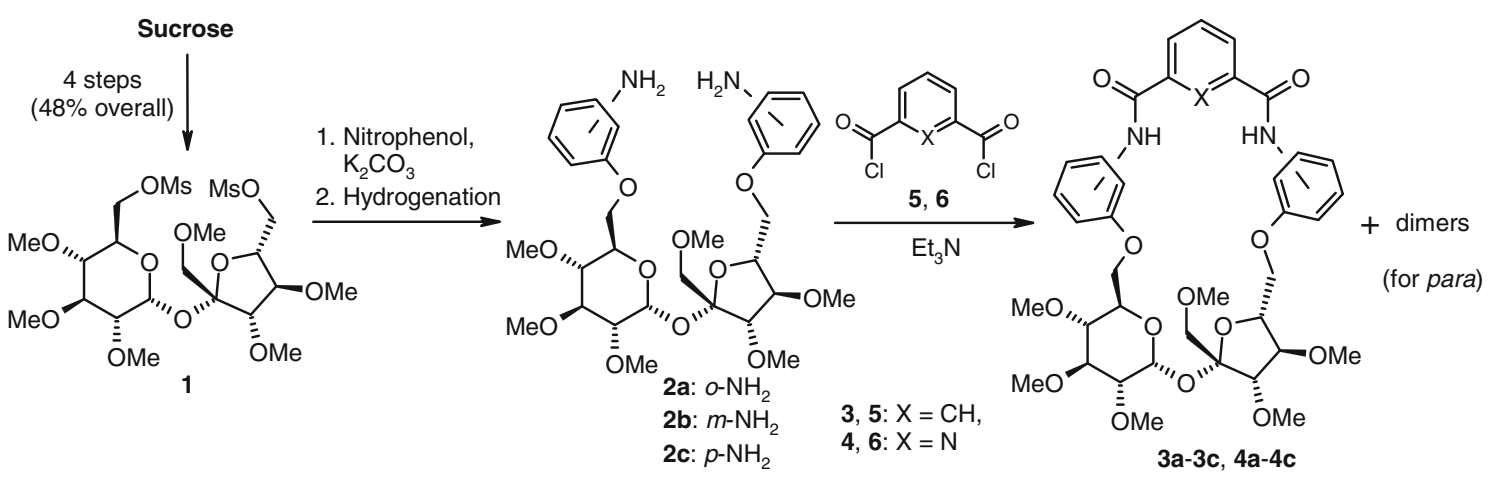

Scheme 1

A crucial aspect of the synthesis of this type of receptor is the relative orientation of the two amino groups in the energetically accessible conformations of the substrates. The amino groups in the $p$-substituted derivative $\mathbf{2 c}$ are rather distant from each other (compared with the $o$ and $m$ analogs $\mathbf{2 a}$ and $\mathbf{2 b}$ ). Thus, the intermediate formed in reaction of the acid dichloride ( $\mathbf{5}$ or $\mathbf{6}$ ) with the first amino group will react preferentially with a second molecule of $\mathbf{2 c}$ (to form the dimer) rather than undergo the intramolecular process leading to $\mathbf{3 c}$ or $\mathbf{4 c}$ [23].

\section{Results and Discussion}

In this paper we report the synthesis of new sucrose macrocyclic derivatives which are twice homologated compared with compounds $3 \mathbf{a}-\mathbf{3 c}$ and $4 \mathbf{a}-\mathbf{4 c}$. Arylmethaneamines 9a-9c (the homologated analogs of anilines 2a-2c) were used as starting materials for the preparation of conformationally less demanding structures.

$1^{\prime}, 2,3,3^{\prime}, 4,4^{\prime}$-Hexa- $O$-methyl-6,6'-di- $O$-(methylsulfonyl)sucrose (1) was treated with 2 equiv. of the appropriate, commercially available cyanophenol $(\mathbf{7 a}-\mathbf{7 c} ; o, m, p$, respectively) in DMF in the presence of potassium carbonate to give the corresponding $6,6^{\prime}$-di- $O$-(cyanophenyl)$1^{\prime}, 2,3,3^{\prime}, 4,4^{\prime}$-hexa- $O$-methylsucroses $(\mathbf{8 a}-\mathbf{8 c})$ in $81-84 \%$ yield. These compounds were quantitatively converted into the 6,6'-di- $O$-[(aminomethyl)phenyl]-1',2,3,3',4,4'-hexa- $O$ methylsucroses $(\mathbf{9 a}-9 \mathbf{c})$ by reduction with $\mathrm{LiAlH}_{4}$. The crude bis-amines 9a-9c were subjected to cyclocondensation reaction with isophthaloyl or 2,6-pyridinedicarbonyl dichlorides ( $\mathbf{5}$ and $\mathbf{6}$, respectively) to achieve closure of the ring (Scheme 2). To avoid formation of the dimeric byproducts, the reactions were performed in dilute solution. In all cases a 1:1-product (10a-10c and 11a-11c) was formed in good yield (63-74 \%; Fig. 1).

In summary, we have developed a simple, rapid, and efficient procedure for preparation of sucrose-based promising optically active receptors. Because of the conformational mobility (less rigid structure) of the diamine $\mathbf{9 c}$, which differ from $2 \mathbf{c}$ (which furnishes both the monomers and the dimers in the reaction with dichlorides $\mathbf{5}$ or $\mathbf{6}$; Scheme 1) only in the length of the chain, we were able to suppress formation of the dimer and obtain monomeric macrocycles in good yield. This strategy was applicable to the synthesis of sucrose-derived macrocycles containing isophthalic and pyridine-2,6-diamide groups.

\section{Experimental}

All reported NMR spectra were recorded with a Varian Vnmrs-600 MHz spectrometer (at 600 and $150 \mathrm{MHz}$ for ${ }^{1} \mathrm{H}$ and ${ }^{13} \mathrm{C}$ NMR spectra, respectively); solutions were prepared in $\mathrm{CDCl}_{3}$ with TMS as the internal standard. Most of the resonances were assigned by $\operatorname{COSY}\left({ }^{1} \mathrm{H}-{ }^{1} \mathrm{H}\right)$ and gradient selected HSQC and HMBC correlations. IR spectra $\left(\mathrm{CHCl}_{3}\right.$, film) were recorded on a Perkin Elmer FTIR Spectrum 2000. Mass spectra were recorded with an ESI/MS Mariner (PerSeptive Biosystem) mass spectrometer. Elemental analysis was performed with a Perkin-Elmer $2400 \mathrm{CHN}$ analyzer; results agreed satisfactorily with calculated values. Optical rotation was measured with a Jasco DIP-360 digital polarimeter; solutions were prepared in $\mathrm{CH}_{2} \mathrm{Cl}_{2}(c=1)$. Flash chromatography was performed on silica gel (Merck, 230-400 mesh). The organic phases were dried over anhydrous magnesium sulfate.

General procedure for preparation of 6, $6^{\prime}$-di-O(cyanophenyl)-1',2,3,3',4,4'-hexa-O-methylsucroses $(8 a-8 c)$

To a solution of $291 \mathrm{mg}$ compound $1(0.5 \mathrm{mmol})$ in $25 \mathrm{~cm}^{3}$ dry DMF, $345 \mathrm{mg} \mathrm{K}_{2} \mathrm{CO}_{3}(2.5 \mathrm{mmol})$ was added than $179 \mathrm{mg}$ of the corresponding cyanophenol 7a-7c $(1.5 \mathrm{mmol})$. The mixture was stirred for $24 \mathrm{~h}$ at $100{ }^{\circ} \mathrm{C}$ then cooled to room temperature. Water $\left(50 \mathrm{~cm}^{3}\right)$ and $50 \mathrm{~cm}^{3}$ AcOEt were added, the organic phase was separated, and the aqueous 


\section{Scheme 2}

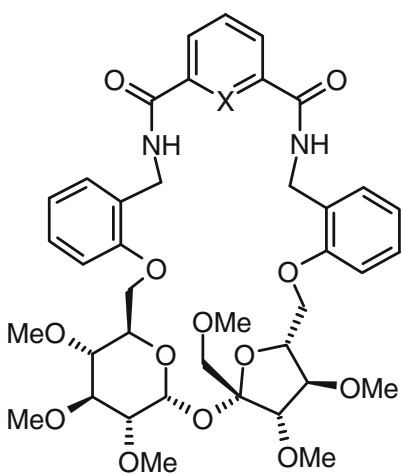

10a: $\mathrm{X}=\mathrm{CH}(64 \%)$

11a: $X=N(67 \%)$<smiles></smiles>

10b: $\mathrm{X}=\mathrm{CH}(63 \%)$

11b: $X=N(66 \%)$

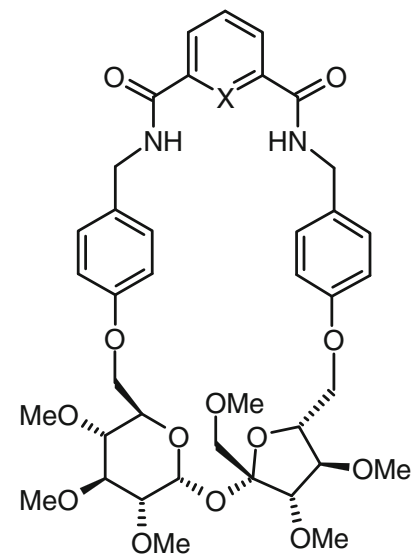

10c: $\mathrm{X}=\mathrm{CH}(71 \%)$ 11c: $X=N(74 \%)$

Fig. 1 Macrocyclic diamides 10a-10c and 11a-11c

phase was extracted with ethyl acetate $\left(4 \times 50 \mathrm{~cm}^{3}\right)$. Combined organic solutions were washed with water $\left(2 \times 30 \mathrm{~cm}^{3}\right), 30 \mathrm{~cm}^{3}$ brine, dried, concentrated, and the product was isolated by flash chromatography (hexane-ethyl acetate, $9: 1$ to $7: 3$ ) to afford $\mathbf{8 a}-\mathbf{8 c}$.
6,6'-Di-O-(2-cyanophenyl)-1',2,3,3',4,4'-hexa-Omethylsucrose (8a, $\mathrm{C}_{32} \mathrm{H}_{40} \mathrm{~N}_{2} \mathrm{O}_{11}$ )

Colorless oil; yield: $255 \mathrm{mg}(81 \%)$; TLC: $R_{f}=0.47$ (hexane/AcOEt 1:2); $[\alpha]_{\mathrm{D}}^{24}=+55.9^{\circ} \mathrm{cm}^{2} \mathrm{~g}^{-1} ;$ IR: $\bar{v}=2,983$, 2,934, 2,832, 2,228, 1,741, 1,599, 1,581, 1,494, 1,449, 1,374, 
$1,292,1,261,1,185,1,164,1,102,1,045,1,018,983,879$, 835, 757, 667, 566, $497 \mathrm{~cm}^{-1}$; ${ }^{1} \mathrm{H}$ NMR: $\delta=7.55(\mathrm{dd}$, $J=7.7 \mathrm{~Hz}, 1.7 \mathrm{~Hz}, 1 \mathrm{H}, \mathrm{Ar}), 7.53(\mathrm{dd}, J=7.5 \mathrm{~Hz}, 1.7 \mathrm{~Hz}$, $1 \mathrm{H}, \mathrm{Ar}$ ), 7.51 (ddd, $J=8.4 \mathrm{~Hz}, 7.6 \mathrm{~Hz}, 1.6 \mathrm{~Hz}, 1 \mathrm{H}, \mathrm{Ar}$ ), 7.37 (ddd, $J=8.5 \mathrm{~Hz}, 7.6 \mathrm{~Hz}, 1.7 \mathrm{~Hz}, 1 \mathrm{H}, \mathrm{Ar}$ ), 6.99-7.07 (m, 3H, Ar), 6.95 (dd, $J=7.6 \mathrm{~Hz}, 7.6 \mathrm{~Hz}, 1 \mathrm{H}, \mathrm{Ar}), 5.59$ (d, $\left.J_{1,2}=3.7 \mathrm{~Hz}, 1 \mathrm{H}, \mathrm{H}-1\right), 4.32-4.38\left(\mathrm{~m}, 2 \mathrm{H}, 2 \mathrm{H}-6^{\prime}\right), 4.23-$ 4.27 (m, 3H, H-5', 2H-6), $4.18(\mathrm{~m}, 1 \mathrm{H}, \mathrm{H}-5), 4.09$ $\left(\mathrm{d}, J_{3^{\prime}, 4^{\prime}}=6.7 \mathrm{~Hz}, 1 \mathrm{H}, \mathrm{H}-3^{\prime}\right), 3.90\left(\mathrm{dd}, J_{4^{\prime}, 3^{\prime}}=6.7 \mathrm{~Hz}\right.$, $\left.J_{4^{\prime}, 5^{\prime}}=6.1 \mathrm{~Hz}, 1 \mathrm{H}, \mathrm{H}-4^{\prime}\right), 3.66\left(\mathrm{~d}, J_{1^{\prime}, 1^{\prime}}=11.1 \mathrm{~Hz}, 1 \mathrm{H}, \mathrm{H}-\right.$ $\left.1^{\prime}\right), 3.60\left(\mathrm{~s}, 3 \mathrm{H}, \mathrm{CH}_{3}\right), 3.54\left(\mathrm{~s}, 3 \mathrm{H}, \mathrm{CH}_{3}\right), 3.51(\mathrm{dd}$, $\left.J_{3,2}=9.7 \mathrm{~Hz}, J_{3,4}=9.1 \mathrm{~Hz}, 1 \mathrm{H}, \mathrm{H}-3\right), 3.49\left(\mathrm{~s}, 3 \mathrm{H}, \mathrm{CH}_{3}\right.$ ), $3.47\left(\mathrm{~s}, \quad 3 \mathrm{H}, \quad \mathrm{CH}_{3}\right), \quad 3.46\left(\mathrm{~s}, \quad 3 \mathrm{H}, \mathrm{CH}_{3}\right), 3.43(\mathrm{~d}$, $\left.J_{1^{\prime}, 1^{\prime}}=11.1 \mathrm{~Hz}, 1 \mathrm{H}, \mathrm{H}-1^{\prime}\right), 3.42\left(\mathrm{~s}, 3 \mathrm{H}, \mathrm{CH}_{3}\right), 3.34(\mathrm{dd}$, $\left.J_{4,3}=9.1 \mathrm{~Hz}, \quad J_{4,5}=10.0 \mathrm{~Hz}, \quad 1 \mathrm{H}, \quad \mathrm{H}-4\right), 3.16 \quad(\mathrm{dd}$, $\left.J_{2,1}=3.7 \mathrm{~Hz}, J_{2,3}=9.7 \mathrm{~Hz}, 1 \mathrm{H}, \mathrm{H}-2\right) \mathrm{ppm} ;{ }^{13} \mathrm{C}$ NMR: $\delta=160.49,160.33,134.33,134.15,133.82,133.82,121.13$, 121.01, 116.40, 116.30, 112.87, 112.79 (12 × C-Ar), 104.98 $\left(\mathrm{C}-2^{\prime}\right), 102.33(\mathrm{CN}), 102.25(\mathrm{CN}), 90.02(\mathrm{C}-1), 85.88\left(\mathrm{C}-3^{\prime}\right)$, 84.92 (C-4'), 83.12 (C-3), 81.46 (C-2), 79.16 (C-4), 78.83 (C-5'), 73.30 (C-1'), 70.42 (C-6'), 69.71 (C-5), 68.52 (C-6), $60.63,60.47,59.51,58.75,58.55,58.46\left(6 \times \mathrm{OCH}_{3}\right) \mathrm{ppm}$; HRMS (ESI): calcd for $\mathrm{C}_{32} \mathrm{H}_{40} \mathrm{~N}_{2} \mathrm{O}_{11} \mathrm{Na} \quad[\mathrm{M}+\mathrm{Na}]^{+}$ 651.2524 , found 651.2525 .

\section{6,6'-Di-O-(3-cyanophenyl)-1',2,3,3',4,4'-hexa-O-}

\section{methylsucrose (8b, $\mathrm{C}_{32} \mathrm{H}_{40} \mathrm{~N}_{2} \mathrm{O}_{11}$ )}

Colorless oil; yield: $265 \mathrm{mg}(84 \%)$; TLC: $R_{f}=0.51$ (hexane/AcOEt $1: 2$ ); $[\alpha]_{\mathrm{D}}^{22}=+56.3^{\circ} \mathrm{cm}^{2} \mathrm{~g}^{-1} ; \quad$ IR: $\bar{v}=3,075,2,982,2,933,2,831,2,231,1,741,1,597$, $1,579,1,483,1,432,1,328,1,291,1,265,1,185,1,148$, 1,101, 1,017, 983, 873, 790, 756, 682, 616, 517, $475 \mathrm{~cm}^{-1}$; ${ }^{1} \mathrm{H}$ NMR: $\delta=7.36(\mathrm{dd}, J=7.8 \mathrm{~Hz}, 8.0 \mathrm{~Hz}, 1 \mathrm{H}, \mathrm{Ar}), 7.28$ $(\mathrm{dd}, J=7.8 \mathrm{~Hz}, 8.2 \mathrm{~Hz}, 1 \mathrm{H}, \mathrm{Ar}), 7.24(\mathrm{~d}, J=7.6 \mathrm{~Hz}, 1 \mathrm{H}$, $\mathrm{Ar}), 7.20(\mathrm{~d}, J=7.4 \mathrm{~Hz}, 1 \mathrm{H}, \mathrm{Ar}), 7.12-7.17$ (m, 3H, Ar), $7.11(\mathrm{dd}, \quad J=8.2 \mathrm{~Hz}, \quad 2.3 \mathrm{~Hz}, \quad 1 \mathrm{H}, \quad \mathrm{Ar}), 5.60$ (d, $\left.J_{1,2}=3.7 \mathrm{~Hz}, 1 \mathrm{H}, \mathrm{H}-1\right), 4.29\left(\mathrm{~m}, 1 \mathrm{H}, \mathrm{H}-6^{\prime}\right), 4.12-4.23$ (m, 5H, H-5, H-5', 2H-6, H-6 $\left.{ }^{\prime}\right), 4.11$ (d, $J_{3^{\prime}, 4^{\prime}}=7.6 \mathrm{~Hz}$, $\left.1 \mathrm{H}, \mathrm{H}-3^{\prime}\right), 3.97\left(\mathrm{dd}, J_{4^{\prime}, 3^{\prime}}=7.6 \mathrm{~Hz}, J_{4^{\prime}, 5^{\prime}}=7.3 \mathrm{~Hz}, 1 \mathrm{H}, \mathrm{H}-\right.$ $\left.4^{\prime}\right), 3.64\left(\mathrm{~s}, 3 \mathrm{H}, \mathrm{CH}_{3}\right), 3.61\left(\mathrm{~d}, J_{1^{\prime}, 1^{\prime}}=11.0 \mathrm{~Hz}, 1 \mathrm{H}, \mathrm{H}-1^{\prime}\right)$, 3.53 (s, $\left.3 \mathrm{H}, \mathrm{CH}_{3}\right), 3.51$ (s, $\left.3 \mathrm{H}, \mathrm{CH}_{3}\right), 3.49(\mathrm{~m}, 1 \mathrm{H}, \mathrm{H}-3)$, 3.47 (s, 3H, $\left.\mathrm{CH}_{3}\right), 3.44$ (s, 3H, $\left.\mathrm{CH}_{3}\right), 3.43\left(\mathrm{~s}, 3 \mathrm{H}, \mathrm{CH}_{3}\right)$, $3.43\left(\mathrm{~d}, J_{1^{\prime}, 1^{\prime}}=11.0 \mathrm{~Hz}, 1 \mathrm{H}, \mathrm{H}-1^{\prime}\right), 3.22(\mathrm{dd}, J=10.2 \mathrm{~Hz}$, $8.9 \mathrm{~Hz}, 1 \mathrm{H}, \mathrm{H}-4), 3.16\left(\mathrm{dd}, J_{2,1}=3.7 \mathrm{~Hz}, J_{2,3}=9.7 \mathrm{~Hz}\right.$, $1 \mathrm{H}, \mathrm{H}-2) \mathrm{ppm} ;{ }^{13} \mathrm{C}$ NMR: $\delta=158.74,158.65,130.47$, $130.26,124.83,124.76,119.90,119.84,118.51,118.51$, 117.48, $117.39(12 \times \mathrm{C}-\mathrm{Ar}), 113.30(\mathrm{CN}), 113.16(\mathrm{CN})$, 104.39 (C-2'), 89.39 (C-1), $85.34\left(\mathrm{C}-3^{\prime}\right), 83.79$ (C-4'), 83.21 (C-3), 81.64 (C-2), 79.47 (C-4), 78.53 (C-5'), 73.70 $\left(\mathrm{C}-1^{\prime}\right), 69.66$ (C-5), 69.30 (C-6'), 67.80 (C-6), 60.76, 60.58, 59.42, 58.65, 58.47, $58.43\left(6 \times \mathrm{OCH}_{3}\right) \mathrm{ppm}$; HRMS (ESI): calcd for $\mathrm{C}_{32} \mathrm{H}_{40} \mathrm{~N}_{2} \mathrm{O}_{11} \mathrm{Na}[\mathrm{M}+\mathrm{Na}]^{+}$651.2524, found 651.2522 .
6,6'-Di-O-(4-cyanophenyl)-1',2,3,3',4,4'-hexa-O-

methylsucrose (8c, $\mathrm{C}_{32} \mathrm{H}_{40} \mathrm{~N}_{2} \mathrm{O}_{11}$ )

Colorless oil; yield: $258 \mathrm{mg}$ (82\%); TLC: $R_{f}=0.54$ (hexane/AcOEt $1: 2$ ); $[\alpha]_{\mathrm{D}}^{24}=+75.8^{\circ} \mathrm{cm}^{2} \mathrm{~g}^{-1} ; \quad$ IR: $\bar{v}=2,983,2,933,2,831,2,225,1,606,1,575,1,509$, $1,453,1,419,1,374,1,302,1,259,1,173,1,150,1,100$, 1,019, 983, 836, 755, 724, 684, $548 \mathrm{~cm}^{-1}$; ${ }^{1} \mathrm{H}$ NMR: $\delta=7.59(\mathrm{~d}, J=9.0 \mathrm{~Hz}, 2 \mathrm{H}, \mathrm{Ar}), 7.50(\mathrm{~d}, J=9.0 \mathrm{~Hz}, 2 \mathrm{H}$, $\mathrm{Ar}), 6.98(\mathrm{~d}, J=9.0 \mathrm{~Hz}, 2 \mathrm{H}, \mathrm{Ar}), 6.94(\mathrm{~d}, J=9.0 \mathrm{~Hz}, 2 \mathrm{H}$, Ar), $5.60\left(\mathrm{~d}, J_{1,2}=3.7 \mathrm{~Hz}, 1 \mathrm{H}, \mathrm{H}-1\right), 4.32\left(\mathrm{~m}, 1 \mathrm{H}, \mathrm{H}-6^{\prime}\right)$, 4.14-4.22 (m, 5H, H-5, 2H-6, H-5', H-6'), 4.10 (d, $\left.J_{3^{\prime}, 4^{\prime}}=7.5 \mathrm{~Hz}, \quad 1 \mathrm{H}, \quad \mathrm{H}-3^{\prime}\right), 3.93\left(\mathrm{dd}, J_{4^{\prime}, 3^{\prime}}=7.5 \mathrm{~Hz}\right.$, $\left.J_{4^{\prime}, 5^{\prime}}=7.3 \mathrm{~Hz}, 1 \mathrm{H}, \mathrm{H}-4^{\prime}\right), 3.62\left(\mathrm{~s}, 3 \mathrm{H}, \mathrm{CH}_{3}\right), 3.61(\mathrm{~d}$, $\left.J_{1^{\prime}, 1^{\prime}}=10.8 \mathrm{~Hz}, 1 \mathrm{H}, \mathrm{H}-1^{\prime}\right), 3.52\left(\mathrm{~s}, 3 \mathrm{H}, \mathrm{CH}_{3}\right), 3.49(\mathrm{~s}, 3 \mathrm{H}$, $\left.\mathrm{CH}_{3}\right), 3.49\left(\mathrm{dd}, J_{3,2}=9.7 \mathrm{~Hz}, J_{3,4}=8.9 \mathrm{~Hz}, 1 \mathrm{H}, \mathrm{H}-3\right)$, $3.435\left(\mathrm{~s}, 3 \mathrm{H}, \mathrm{CH}_{3}\right), 3.432\left(\mathrm{~s}, 3 \mathrm{H}, \mathrm{CH}_{3}\right), 3.427(\mathrm{~d}$, $\left.J_{1^{\prime}, 1^{\prime}}=10.8 \mathrm{~Hz}, 1 \mathrm{H}, \mathrm{H}-1^{\prime}\right), 3.418\left(\mathrm{~s}, 3 \mathrm{H}, \mathrm{CH}_{3}\right), 3.20(\mathrm{dd}$, $\left.J_{4,3}=8.9 \mathrm{~Hz}, \quad J_{4,5}=9.7 \mathrm{~Hz}, \quad 1 \mathrm{H}, \quad \mathrm{H}-4\right), 3.15 \quad(\mathrm{dd}$, $\left.J_{2,1}=3.7 \mathrm{~Hz}, J_{2,3}=9.7 \mathrm{~Hz}, 1 \mathrm{H}, \mathrm{H}-2\right) \mathrm{ppm} ;{ }^{13} \mathrm{C}$ NMR: $\delta=161.92,161.89,134.11,133.93,119.03,118.94$, 115.33, $115.27(12 \times \mathrm{C}-\mathrm{Ar}), 104.54(\mathrm{CN}), 104.50\left(\mathrm{C}-2^{\prime}\right)$, $104.36(\mathrm{CN}), 89.46(\mathrm{C}-1), 85.35\left(\mathrm{C}-3^{\prime}\right), 83.64\left(\mathrm{C}-4^{\prime}\right), 83.24$ (C-3), 81.70 (C-2), 79.47 (C-4), 78.49 (C-5'), 73.68 (C-1'), 69.51 (C-5), 69.33 (C-6'), 67.78 (C-6), 60.78, 60.63, 59.46, 58.72, 58.51, $58.39\left(6 \times \mathrm{OCH}_{3}\right)$ ppm; HRMS (ESI): calcd for $\mathrm{C}_{32} \mathrm{H}_{40} \mathrm{~N}_{2} \mathrm{O}_{11} \mathrm{Na}[\mathrm{M}+\mathrm{Na}]^{+}$651.2524, found 651.2538 .

General procedure for synthesis of 6,6'-di-O[4-(aminomethyl)phenyl]-1',2,3,3',4,4'-hexa-Omethylsucroses $(\mathbf{9 a}-\mathbf{9 c})$

To a cooled $\left(0{ }^{\circ} \mathrm{C}\right)$ solution of $215 \mathrm{mg}$ compound $\mathbf{8 a}-\mathbf{8 c}$ $(0.34 \mathrm{mmol})$ in $30 \mathrm{~cm}^{3}$ dry THF, $93 \mathrm{mg} \mathrm{LiAlH}_{4}$ (2.45 mmol) was added slowly within $5 \mathrm{~min}$. The mixture was stirred for $1 \mathrm{~h}$ at $60{ }^{\circ} \mathrm{C}$ and cooled to room temperature. Excess hydride was carefully decomposed with $10 \mathrm{~cm}^{3}$ water and $40 \mathrm{~cm}^{3}$ aqueous potassium bisulfate $\left(\mathrm{KHSO}_{4}\right)$. Ethyl acetate $\left(50 \mathrm{~cm}^{3}\right)$ was added, the layers were separated, and the aqueous layer was extracted with ethyl acetate $\left(3 \times 40 \mathrm{~cm}^{3}\right)$. The combined organic solutions were dried, concentrated, and the crude product was used in the next step without purification.

\section{General procedure for syntheses of macrocyclic diamides 10a-10c and 11a-11c}

This reaction was conducted under an argon atmosphere: $35 \mathrm{mg}$ isophthaloyl or 2,6-pyridinedicarbonyl dichloride (5 or $6,0.17 \mathrm{mmol}$ ) was dissolved in $20 \mathrm{~cm}^{3}$ dry $\mathrm{CH}_{2} \mathrm{Cl}_{2}$ and added dropwise to a stirred solution of $108 \mathrm{mg}$ diamine 9a-9c $(0.17 \mathrm{mmol})$ in $40 \mathrm{~cm}^{3}$ dry $\mathrm{CH}_{2} \mathrm{Cl}_{2}$ containing $71 \mathrm{~mm}^{3} \mathrm{Et}_{3} \mathrm{~N}(0.51 \mathrm{mmol})$, and the mixture was stirred for 
$1 \mathrm{~h}$ at room temperature. The resulting solution was concentrated in vacuum and the residue was dissolved in $40 \mathrm{~cm}^{3}$ ethyl acetate and $20 \mathrm{~cm}^{3}$ water. Saturated $\mathrm{K}_{2} \mathrm{CO}_{3}$ solution $\left(10 \mathrm{~cm}^{3}\right)$ was added, the layers were separated, and the aqueous layer was extracted with ethyl acetate $\left(3 \times 30 \mathrm{~cm}^{3}\right)$. The combined organic extracts were washed with $20 \mathrm{~cm}^{3}$ water and $10 \mathrm{~cm}^{3}$ brine, dried, concentrated, and the products were isolated by flash chromatography (hexane-ethyl acetate, 50:50 to 25:75).

6,6'-Di-O-[[benzene-1,3-diyl-bis(carbonylaminomethyl)]2,2'-diphenyl]-1',2,3,3',4,4'-hexa-O-methylsucrose

(10a, $\mathrm{C}_{40} \mathrm{H}_{50} \mathrm{~N}_{2} \mathrm{O}_{13}$ )

White solid; yield: $84 \mathrm{mg}$ (64\%); m.p.: $134{ }^{\circ} \mathrm{C}$; TLC: $R_{f}=0.35 \quad(\mathrm{AcOEt}) ; \quad[\alpha]_{\mathrm{D}}^{22}=+78.8^{\circ} \mathrm{cm}^{2} \mathrm{~g}^{-1} ; \quad$ IR: $\bar{v}=3,347,3,064,2,982,2,933,2,830,1,658,1,603$, $1,590,1,526,1,495,1,451,1,359,1,318,1,293,1,250$, $1,186,1,161,1,100,1,049,1,017,1,004,982,941,882$, 825, 753, 710, 593, $527 \mathrm{~cm}^{-1} ;{ }^{1} \mathrm{H}$ NMR: $\delta=8.01-8.05$ $(\mathrm{m}, 2 \mathrm{H}$, isophthalic), $7.57(\mathrm{~s}, 1 \mathrm{H}$, isophthalic), $7.52(\mathrm{t}$, $J=7.7 \mathrm{~Hz}, 1 \mathrm{H}$, isophthalic), $7.32(\mathrm{~d}, J=7.3 \mathrm{~Hz}, 1 \mathrm{H}, \mathrm{Ar})$, 7.26-7.31 (m, 3H, Ar), 6.92-6.96 (m, 2H, Ar), 6.90 $(\mathrm{d}, J=8.5 \mathrm{~Hz}, 1 \mathrm{H}, \mathrm{Ar}), 6.85(\mathrm{br} \mathrm{s}, 1 \mathrm{H}, \mathrm{NH}), 6.74$ (d, $J=8.0 \mathrm{~Hz}, 1 \mathrm{H}, \mathrm{Ar}$ ), 6.66 (br s, $1 \mathrm{H}, \mathrm{NH}), 4.72-4.77$ $\left(\mathrm{m}, 2 \mathrm{H}, \mathrm{CH}_{2} \mathrm{~N}\right), 4.59\left(\mathrm{~d}, J_{1,2}=3.3 \mathrm{~Hz}, 1 \mathrm{H}, \mathrm{H}-1\right), 4.51(\mathrm{dd}$, $\left.J=13.8 \mathrm{~Hz}, 6.2 \mathrm{~Hz}, 1 \mathrm{H}, \mathrm{CH}_{2} \mathrm{~N}\right), 4.45(\mathrm{dd}, J=13.6 \mathrm{~Hz}$, $\left.6.5 \mathrm{~Hz}, 1 \mathrm{H}, \mathrm{CH}_{2} \mathrm{~N}\right), 4.27\left(\mathrm{dd}, J_{6^{\prime}, 6^{\prime}}=9.9 \mathrm{~Hz}, J_{6^{\prime}, 5^{\prime}}=\right.$ $\left.2.3 \mathrm{~Hz}, 1 \mathrm{H}, \mathrm{H}-6^{\prime}\right), 4.14-4.20$ (m, 2H, H-5', H-6), 4.08 (d, $\left.J_{3^{\prime}, 4^{\prime}}=7.4 \mathrm{~Hz}, 1 \mathrm{H}, \mathrm{H}-3^{\prime}\right), 3.93-3.97\left(\mathrm{~m}, 2 \mathrm{H}, \mathrm{H}-5, \mathrm{H}-6^{\prime}\right)$, $3.76\left(\mathrm{dd}, J_{6,6}=10.0 \mathrm{~Hz}, J_{6,5}=1.5 \mathrm{~Hz}, 1 \mathrm{H}, \mathrm{H}-6\right), 3.70$ $\left(\mathrm{dd}, J_{4^{\prime}, 5^{\prime}}=7.5 \mathrm{~Hz}, J_{4^{\prime}, 3^{\prime}}=7.4 \mathrm{~Hz}, 1 \mathrm{H}, \mathrm{H}-4^{\prime}\right), 3.56(\mathrm{~s}, 3 \mathrm{H}$, $\left.\mathrm{CH}_{3}\right), 3.47$ (s, $\left.3 \mathrm{H}, \mathrm{CH}_{3}\right), 3.46(\mathrm{~m}, 1 \mathrm{H}, \mathrm{H}-3), 3.440$ (s, $3 \mathrm{H}$, $\mathrm{CH}_{3}$ ), 3.435 (s, 3H, $\mathrm{CH}_{3}$ ), 3.40-3.43 (m, 2H, H-1', H-4), $3.39\left(\mathrm{~s}, 3 \mathrm{H}, \mathrm{CH}_{3}\right), 3.23\left(\mathrm{~s}, 3 \mathrm{H}, \mathrm{CH}_{3}\right), 3.14\left(\mathrm{~d}, J_{1^{\prime}, 1^{\prime}}=\right.$ $\left.11.2 \mathrm{~Hz}, 1 \mathrm{H}, \mathrm{H}-1^{\prime}\right), 2.75\left(\mathrm{dd}, J_{2,1}=3.3 \mathrm{~Hz}, J_{2,3}=9.5 \mathrm{~Hz}\right.$, $1 \mathrm{H}, \mathrm{H}-2)$ ppm; ${ }^{13} \mathrm{C}$ NMR: $\delta=166.96(\mathrm{C}=\mathrm{O}), 166.75$ $(\mathrm{C}=\mathrm{O}), \quad 156.85, \quad 156.81 \quad(2 \times \mathrm{C}-\mathrm{Ar}), \quad 135.53, \quad 135.42$, $131.21, \quad 130.87$ (4 $\times$ C-isophthalic $), \quad 131.09, \quad 130.84$, $129.43, \quad 129.31 \quad(4 \times \mathrm{C}-\mathrm{Ar}), \quad 129.16$ (C-isophthalic), $127.05, \quad 125.73 \quad(2 \times \mathrm{C}-\mathrm{Ar}), \quad 123.80 \quad$ (C-isophthalic), $121.72,121.10,112.51,110.99(4 \times \mathrm{C}-\mathrm{Ar}), 104.37(\mathrm{C}-$ 2'), 90.41 (C-1), 84.70 (C-3'), 84.09 (C-4'), 82.72 (C-3),

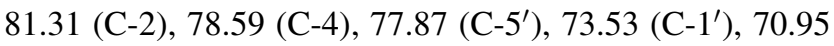
(C-6'), 70.25 (C-5), 66.07 (C-6), 60.67, 60.33, 59.67, 58.87, 58.04, $57.99\left(6 \times \mathrm{OCH}_{3}\right), 41.53,40.94\left(2 \times \mathrm{CH}_{2} \mathrm{~N}\right) \mathrm{ppm}$; HRMS (ESI): calcd for $\mathrm{C}_{40} \mathrm{H}_{50} \mathrm{~N}_{2} \mathrm{O}_{13} \mathrm{Na}[\mathrm{M}+\mathrm{Na}]^{+}$ 789.3205, found 789.3228 .

6,6'-Di-O-[[pyridine-1,3-diyl-bis(carbonylaminomethyl)]2,2'-diphenyl]-1',2,3,3',4,4'-hexa-O-methylsucrose

(11a, $\mathrm{C}_{39} \mathrm{H}_{49} \mathrm{~N}_{3} \mathrm{O}_{13}$ )

White solid; yield: $88 \mathrm{mg}(67 \%)$; m.p.: $96{ }^{\circ} \mathrm{C}$; TLC: $R_{f}=0.37 \quad(\mathrm{AcOEt}) ; \quad[\alpha]_{\mathrm{D}}^{22}=+163.1^{\circ} \mathrm{cm}^{2} \mathrm{~g}^{-1} ; \quad$ IR: $\bar{v}=3,537,3,403,3,303,3,064,2,984,2,933,2,831$,
$1,735,1,674,1,602,1,590,1,528,1,494,1,452,1,360$, $1,289,1,278,1,244,1,186,1,161,1,149,1,101,1,051$, $1,017,1,003,983,945,878,844,754,683,647,609$, $564 \mathrm{~cm}^{-1}$; ${ }^{1} \mathrm{H}$ NMR: $\delta=8.48(\mathrm{dd}, J=4.3 \mathrm{~Hz}, 7.3 \mathrm{~Hz}$, $1 \mathrm{H}, \quad \mathrm{NH}), \quad 8.34-8.37(\mathrm{~m}, 2 \mathrm{H}$, pyridine $), 8.22(\mathrm{dd}$, $J=4.1 \mathrm{~Hz}, 7.6 \mathrm{~Hz}, 1 \mathrm{H}, \mathrm{NH}), 8.02(\mathrm{t}, J=7.8 \mathrm{~Hz}, 1 \mathrm{H}$, pyridine), 7.39 (dd, $J=7.5 \mathrm{~Hz}, 1.6 \mathrm{~Hz}, 1 \mathrm{H}, \mathrm{Ar}), 7.30$ (dd, $J=7.6 \mathrm{~Hz}, 1.6 \mathrm{~Hz}, 1 \mathrm{H}, \mathrm{Ar}), 7.23-7.29$ (m, 2H, Ar), 6.99 $(\mathrm{dd}, J=8.2 \mathrm{~Hz}, 0.8 \mathrm{~Hz}, 1 \mathrm{H}, \mathrm{Ar}), 6.93(\mathrm{ddd}, J=7.5 \mathrm{~Hz}$, $7.4 \mathrm{~Hz}, 0.9 \mathrm{~Hz}, 1 \mathrm{H}, \mathrm{Ar}), 6.88$ (ddd, $J=7.6 \mathrm{~Hz}, 7.4 \mathrm{~Hz}$, $0.8 \mathrm{~Hz}, 1 \mathrm{H}, \mathrm{Ar}), 6.99(\mathrm{dd}, J=8.2 \mathrm{~Hz}, 0.9 \mathrm{~Hz}, 1 \mathrm{H}, \mathrm{Ar})$, $5.35\left(\mathrm{~d}, J_{1,2}=3.4 \mathrm{~Hz}, 1 \mathrm{H}, \mathrm{H}-1\right), 4.85(\mathrm{dd}, J=14.4 \mathrm{~Hz}$, $\left.4.3 \mathrm{~Hz}, 1 \mathrm{H}, \mathrm{CH}_{2} \mathrm{~N}\right), 4.78(\mathrm{dd}, J=14.2 \mathrm{~Hz}, 4.1 \mathrm{~Hz}, 1 \mathrm{H}$, $\left.\mathrm{CH}_{2} \mathrm{~N}\right), 4.69\left(\mathrm{dd}, J=14.4 \mathrm{~Hz}, 7.3 \mathrm{~Hz}, 1 \mathrm{H}, \mathrm{CH}_{2} \mathrm{~N}\right), 4.61$ $\left(\mathrm{dd}, \quad J=14.2 \mathrm{~Hz}, \quad 7.6 \mathrm{~Hz}, \quad 1 \mathrm{H}, \quad \mathrm{CH}_{2} \mathrm{~N}\right), 4.34 \quad(\mathrm{dd}$, $\left.J_{6^{\prime}, 6^{\prime}}=10.2 \mathrm{~Hz}, J_{6^{\prime}, 5^{\prime}}=3.1 \mathrm{~Hz}, 1 \mathrm{H}, \mathrm{H}-6^{\prime}\right), 4.22(\mathrm{~m}, 1 \mathrm{H}$, $\left.\mathrm{H}-5^{\prime}\right), 4.11\left(\mathrm{~d}, J_{3^{\prime}, 4^{\prime}}=6.9 \mathrm{~Hz}, 1 \mathrm{H}, \mathrm{H}-3^{\prime}\right), 4.01-4.07(\mathrm{~m}$, $2 \mathrm{H}, \mathrm{H}-5, \mathrm{H}-6), 3.98\left(\mathrm{dd}, J_{6^{\prime}, 5^{\prime}}=7.7 \mathrm{~Hz}, J_{6^{\prime}, 6^{\prime}}=10.2 \mathrm{~Hz}\right.$, $\left.1 \mathrm{H}, \mathrm{H}-6^{\prime}\right), 3.86\left(\mathrm{dd}, J_{4^{\prime}, 3^{\prime}}=6.9 \mathrm{~Hz}, J_{4^{\prime}, 5^{\prime}}=6.9 \mathrm{~Hz}, 1 \mathrm{H}, \mathrm{H}-\right.$ $\left.4^{\prime}\right), \quad 3.76\left(\mathrm{~d}, \quad J_{6,6}=9.2 \mathrm{~Hz}, \quad 1 \mathrm{H}, \quad \mathrm{H}-6\right), \quad 3.57 \quad(\mathrm{~d}$, $\left.J_{1^{\prime}, 1^{\prime}}=11.0 \mathrm{~Hz}, 1 \mathrm{H}, \mathrm{H}-1^{\prime}\right), 3.52\left(\mathrm{~s}, 3 \mathrm{H}, \mathrm{CH}_{3}\right), 3.48(\mathrm{dd}$, $\left.J_{3,2}=9.6 \mathrm{~Hz}, J_{3,4}=9.0 \mathrm{~Hz}, 1 \mathrm{H}, \mathrm{H}-3\right), 3.460(\mathrm{~s}, 3 \mathrm{H}$, $\left.\mathrm{CH}_{3}\right), 3.457$ (s, $\left.3 \mathrm{H}, \mathrm{CH}_{3}\right), 3.450\left(\mathrm{~s}, 3 \mathrm{H}, \mathrm{CH}_{3}\right), 3.42(\mathrm{~s}, 3 \mathrm{H}$, $\left.\mathrm{CH}_{3}\right), 3.41\left(\mathrm{~d}, J_{1^{\prime}, 1^{\prime}}=11.0 \mathrm{~Hz}, 1 \mathrm{H}, \mathrm{H}-1^{\prime}\right), 3.32(\mathrm{dd}$, $\left.J_{4,3}=9.0 \mathrm{~Hz}, J_{4,5}=9.6 \mathrm{~Hz}, 1 \mathrm{H}, \mathrm{H}-4\right), 3.25(\mathrm{~s}, 3 \mathrm{H}$, $\left.\mathrm{CH}_{3}\right), 2.81\left(\mathrm{dd}, J_{2,1}=3.4 \mathrm{~Hz}, J_{2,3}=9.6 \mathrm{~Hz}, 1 \mathrm{H}, \mathrm{H}-2\right)$ ppm; ${ }^{13} \mathrm{C}$ NMR: $\delta=163.38(\mathrm{C}=\mathrm{O}), 163.12(\mathrm{C}=\mathrm{O}), 156.67$, $156.64(2 \times \mathrm{C}-\mathrm{Ar}), 148.98,148.89,138.83(3 \times$ C-pyridine), 130.72, 130.02, 129.03, 128.71, 128.34, 126.42 $(6 \times \mathrm{C}-\mathrm{Ar}), 124.74,124.74$ ( $2 \times \mathrm{C}-$ pyridine $), 122.67$, 121.43, 114.94, $112.44(4 \times \mathrm{C}-\mathrm{Ar}), 104.92\left(\mathrm{C}-2^{\prime}\right), 89.89$ (C-1), 84.85 (C-3'), 84.19 (C-4'), 82.81 (C-3), 81.88 (C-2),

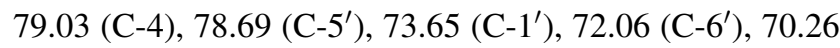
(C-5), 67.30 (C-6), 60.48, 60.42, 59.54, 58.50, 58.38, 58.03 $\left(6 \times \mathrm{OCH}_{3}\right), \quad 39.65,38.14\left(2 \times \mathrm{CH}_{2} \mathrm{~N}\right) \quad$ ppm; HRMS (ESI): calcd for $\mathrm{C}_{39} \mathrm{H}_{49} \mathrm{~N}_{3} \mathrm{O}_{13} \mathrm{Na}[\mathrm{M}+\mathrm{Na}]^{+} 790.3158$, found 790.3165 .

6,6'-Di-O-[[benzene-1,3-diyl-bis(carbonylaminomethyl)]3,3'-diphenyl]-1',2,3,3',4,4'-hexa-O-methylsucrose

(10b, $\mathrm{C}_{40} \mathrm{H}_{50} \mathrm{~N}_{2} \mathrm{O}_{13}$ )

White solid; yield: $82 \mathrm{mg}$ (63\%); m.p.: $111^{\circ} \mathrm{C}$; TLC: $R_{f}=0.36 \quad(\mathrm{AcOEt}) ; \quad[\alpha]_{\mathrm{D}}^{22}=+59.8^{\circ} \mathrm{cm}^{2} \mathrm{~g}^{-1} ; \quad$ IR: $\bar{v}=3,333 ， 2,981,2,931,2,830,1,654,1,599,1,586$, $1,535,1,487,1,448,1,358,1,290,1,267,1,237,1,183$, $1,151,1,100,1,056,1,017,997,983,956,876,755,691$, $622 \mathrm{~cm}^{-1}$; ${ }^{1} \mathrm{H}$ NMR: $\delta=7.97-8.01(\mathrm{~m}, 2 \mathrm{H}$, isophthalic), 7.91 (s, $1 \mathrm{H}$, isophthalic), 7.52 (dd, $J=7.8 \mathrm{~Hz}, 7.8 \mathrm{~Hz}, 1 \mathrm{H}$, isophthalic), 7.24 (dd, $J=7.8 \mathrm{~Hz}, 7.9 \mathrm{~Hz}, 1 \mathrm{H}, \mathrm{Ar}), 7.19$ (dd, $J=7.8 \mathrm{~Hz}, 8.0 \mathrm{~Hz}, 1 \mathrm{H}, \mathrm{Ar}), 6.97$ (s, 1H, Ar), 6.91 (d, $J=7.8 \mathrm{~Hz}, 1 \mathrm{H}, \mathrm{Ar}), 6.87-6.89$ (m, 2H, Ar), 6.82-6.86 (m, $2 \mathrm{H}, \mathrm{Ar}), 6.69(\mathrm{dd}, J=5.6 \mathrm{~Hz}, 5.6 \mathrm{~Hz}, 1 \mathrm{H}, \mathrm{NH}), 6.64(\mathrm{dd}$, $J=5.6 \mathrm{~Hz}, 5.6 \mathrm{~Hz}, 1 \mathrm{H}, \mathrm{NH}), 5.70\left(\mathrm{~d}, J_{1,2}=3.8 \mathrm{~Hz}, 1 \mathrm{H}\right.$, 
$\mathrm{H}-1), 4.65\left(\mathrm{~m}, 2 \mathrm{H}, \mathrm{CH}_{2} \mathrm{~N}\right), 4.50(\mathrm{dd}, J=14.7 \mathrm{~Hz}, 5.6 \mathrm{~Hz}$, $\left.1 \mathrm{H}, \mathrm{CH}_{2} \mathrm{~N}\right), 4.42\left(\mathrm{dd}, J=14.5 \mathrm{~Hz}, 5.6 \mathrm{~Hz}, 1 \mathrm{H}, \mathrm{CH}_{2} \mathrm{~N}\right)$, 4.12-4.23 (m, 4H, H-5, H-6, $\left.2 \times \mathrm{H}^{\prime} 6^{\prime}\right), 4.06-4.12(\mathrm{~m}, 1 \mathrm{H}$, $\left.\mathrm{H}-5^{\prime}\right), 4.09\left(\mathrm{~d}, J_{3^{\prime}, 4^{\prime}}=8.0 \mathrm{~Hz}, 1 \mathrm{H}, \mathrm{H}-3^{\prime}\right), 4.03(\mathrm{br} \mathrm{d}$, $\left.J_{6,6}=9.1 \mathrm{~Hz}, \quad 1 \mathrm{H}, \quad \mathrm{H}-6\right), 3.97 \quad\left(\mathrm{dd}, \quad J_{4^{\prime}, 3^{\prime}}=8.0 \mathrm{~Hz}\right.$, $\left.J_{4^{\prime}, 5^{\prime}}=8.0 \mathrm{~Hz}, 1 \mathrm{H}, \mathrm{H}-4^{\prime}\right), 3.61\left(\mathrm{~s}, 3 \mathrm{H}, \mathrm{CH}_{3}\right), 3.58(\mathrm{~d}$, $\left.J_{1^{\prime}, 1^{\prime}}=10.9 \mathrm{~Hz}, 1 \mathrm{H}, \mathrm{H}-1^{\prime}\right), 3.49\left(\mathrm{~s}, 3 \mathrm{H}, \mathrm{CH}_{3}\right), 3.48(\mathrm{dd}$, $\left.J_{3,2}=9.6 \mathrm{~Hz}, J_{3,4}=9.3 \mathrm{~Hz}, 1 \mathrm{H}, \mathrm{H}-3\right), 3.45\left(\mathrm{~s}, 3 \mathrm{H}, \mathrm{CH}_{3}\right)$, $3.42\left(\mathrm{~d}, J_{1^{\prime}, 1^{\prime}}=10.9 \mathrm{~Hz}, 1 \mathrm{H}, \mathrm{H}-1^{\prime}\right), 3.41\left(\mathrm{~s}, 6 \mathrm{H}, 2 \mathrm{CH}_{3}\right)$, $3.40\left(\mathrm{~s}, 3 \mathrm{H}, \mathrm{CH}_{3}\right), 3.36\left(\mathrm{dd}, J_{4,3}=9.3 \mathrm{~Hz}, J_{4,5}=9.6 \mathrm{~Hz}\right.$, $1 \mathrm{H}, \mathrm{H}-4), 3.21$ (dd, $J_{2,1}=3.8 \mathrm{~Hz}, J_{2,3}=9.6 \mathrm{~Hz}, 1 \mathrm{H}, \mathrm{H}-2$ ) ppm; ${ }^{13} \mathrm{C}$ NMR: $\delta=166.71(\mathrm{C}=\mathrm{O}), 166.34(\mathrm{C}=\mathrm{O}), 159.20$, 159.01, 139.63, 139.28 (4 × C-Ar $), \quad 134.69, \quad 134.56$ (2× C-isophthalic), 130.78 (2C-isophthalic), 129.79, $129.77(2 \times \mathrm{C}-\mathrm{Ar}), 129.39,124.05(2 \times \mathrm{C}$-isophthalic $)$, 121.64, 120.80, 115.13, 114.48, 113.75, 112.49 $(6 \times \mathrm{C}-\mathrm{Ar}), 104.15\left(\mathrm{C}-2^{\prime}\right), 88.69(\mathrm{C}-1), 85.02\left(\mathrm{C}-3^{\prime}\right)$, 83.17 (C-3), 83.03 (C-4'), 81.29 (C-2), 79.14 (C-4), 78.08 $\left(\mathrm{C}^{\prime} 5^{\prime}\right), 74.83\left(\mathrm{C}-1^{\prime}\right), 69.66(\mathrm{C}-5), 68.95\left(\mathrm{C}-6^{\prime}\right), 66.30(\mathrm{C}-6)$, $60.65,60.46,59.41,58.59,58.39,58.03\left(6 \times \mathrm{OCH}_{3}\right)$, 44.25, $43.95\left(2 \times \mathrm{CH}_{2} \mathrm{~N}\right)$ ppm; HRMS (ESI): calcd for $\mathrm{C}_{40} \mathrm{H}_{50} \mathrm{~N}_{2} \mathrm{O}_{13} \mathrm{Na}[\mathrm{M}+\mathrm{Na}]^{+} 789.3202$, found 789.3214 .

6,6'-Di-O-[[pyridine-1,3-diyl-bis(carbonylaminomethyl)]3,3'-diphenyl]-1',2,3,3',4,4'-hexa-O-methylsucrose

$\left(11 b, \mathrm{C}_{39} \mathrm{H}_{49} \mathrm{~N}_{3} \mathrm{O}_{13}\right)$

White solid; yield: $86 \mathrm{mg}(66 \%)$; m.p.: $125^{\circ} \mathrm{C}$; TLC: $R_{f}=0.39 \quad(\mathrm{AcOEt}) ; \quad[\alpha]_{\mathrm{D}}^{22}=+61.6^{\circ} \mathrm{cm}^{2} \mathrm{~g}^{-1} ; \quad$ IR: $\bar{v}=3,317,2,980,2,930,2,831,1,679,1,661,1,599$, $1,586,1,532,1,488,1,448,1,358,1,312,1,287,1,271$, $1,237,1,180,1,148,1,101,1,057,1,038,1,019,1,002,982$, $876,844,755,682,647,623 \mathrm{~cm}^{-1}$; ${ }^{1} \mathrm{H}$ NMR: $\delta=8.34-$ $8.38(\mathrm{~m}, 2 \mathrm{H}$, pyridine), $8.04(\mathrm{t}, J=7.8 \mathrm{~Hz}, 1 \mathrm{H}$, pyridine), $7.90(\mathrm{dd}, J=5.1 \mathrm{~Hz}, 6.6 \mathrm{~Hz}, 1 \mathrm{H}, \mathrm{NH}), 7.85(\mathrm{dd}$, $J=5.6 \mathrm{~Hz}, 5.6 \mathrm{~Hz}, 1 \mathrm{H}, \mathrm{NH}), 7.25(\mathrm{dd}, J=7.5 \mathrm{~Hz}$, $7.9 \mathrm{~Hz}, 1 \mathrm{H}, \mathrm{Ar}), 7.10$ (dd, $J=7.9 \mathrm{~Hz}, 7.9 \mathrm{~Hz}, 1 \mathrm{H}, \mathrm{Ar}$ ), $6.92(\mathrm{~d}, J=7.5 \mathrm{~Hz}, 1 \mathrm{H}, \mathrm{Ar}), 6.91(\mathrm{~d}, J=7.9 \mathrm{~Hz}, 1 \mathrm{H}, \mathrm{Ar})$, 6.81-6.89 (m, 4H, Ar), 5.59 (d, $\left.J_{1,2}=3.7 \mathrm{~Hz}, 1 \mathrm{H}, \mathrm{H}-1\right)$, $4.70\left(\mathrm{dd}, J=14.7 \mathrm{~Hz}, 6.6 \mathrm{~Hz}, 1 \mathrm{H}, \mathrm{CH}_{2} \mathrm{~N}\right), 4.61(\mathrm{dd}$, $\left.J=14.7 \mathrm{~Hz}, 5.6 \mathrm{~Hz}, 1 \mathrm{H}, \mathrm{CH}_{2} \mathrm{~N}\right), 4.58(\mathrm{dd}, J=14.7 \mathrm{~Hz}$, $\left.5.6 \mathrm{~Hz}, 1 \mathrm{H}, \mathrm{CH}_{2} \mathrm{~N}\right), 4.44(\mathrm{dd}, J=14.7 \mathrm{~Hz}, 5.1 \mathrm{~Hz}, 1 \mathrm{H}$, $\left.\mathrm{CH}_{2} \mathrm{~N}\right), 4.27\left(\mathrm{~m}, 1 \mathrm{H}, \mathrm{H}-6^{\prime}\right), 4.21\left(\mathrm{ddd}, J_{5,4}=10.1 \mathrm{~Hz}\right.$, $\left.J_{5,6}=3.9 \mathrm{~Hz}, J_{5,6}=1.6 \mathrm{~Hz}, 1 \mathrm{H}, \mathrm{H}-5\right), 4.10-4.17(\mathrm{~m}, 3 \mathrm{H}$, H-5', H-6, H-6'), 4.08 (d, $\left.J_{3^{\prime}, 4^{\prime}}=7.7 \mathrm{~Hz}, 1 \mathrm{H}, \mathrm{H}-3^{\prime}\right), 4.05$ $\left(\mathrm{dd}, J_{6,6}=10.2 \mathrm{~Hz}, J_{6,5}=3.9 \mathrm{~Hz}, 1 \mathrm{H}, \mathrm{H}-6\right), 3.90(\mathrm{dd}$, $\left.J_{4^{\prime}, 3^{\prime}}=7.7 \mathrm{~Hz}, J_{4^{\prime}, 5^{\prime}}=7.7 \mathrm{~Hz}, 1 \mathrm{H}, \mathrm{H}-4^{\prime}\right), 3.64(\mathrm{~s}, 3 \mathrm{H}$, $\left.\mathrm{CH}_{3}\right), \quad 3.58\left(\mathrm{~d}, \quad J_{1^{\prime}, 1^{\prime}}=11.0 \mathrm{~Hz}, \quad 1 \mathrm{H}, \quad \mathrm{H}-1^{\prime}\right), \quad 3.52$ $\left(\mathrm{dd}, J_{3,2}=9.4 \mathrm{~Hz}, J_{3,4}=9.2 \mathrm{~Hz}, 1 \mathrm{H}, \mathrm{H}-3\right), 3.50(\mathrm{~s}, 3 \mathrm{H}$, $\left.\mathrm{CH}_{3}\right), 3.48\left(\mathrm{~s}, 3 \mathrm{H}, \mathrm{CH}_{3}\right), 3.45\left(\mathrm{~s}, 3 \mathrm{H}, \mathrm{CH}_{3}\right), 3.41$ (s, $\left.3 \mathrm{H}, \mathrm{CH}_{3}\right), 3.41\left(\mathrm{~d}, J_{1^{\prime}, 1^{\prime}}=11.0 \mathrm{~Hz}, 1 \mathrm{H}, \mathrm{H}-1^{\prime}\right), 3.34(\mathrm{~s}, 3 \mathrm{H}$, $\left.\mathrm{CH}_{3}\right), 3.29\left(\mathrm{dd}, J_{4,3}=9.2 \mathrm{~Hz}, J_{4,5}=10.1 \mathrm{~Hz}, 1 \mathrm{H}, \mathrm{H}-4\right)$, $3.19\left(\mathrm{dd}, J_{2,1}=3.7 \mathrm{~Hz}, J_{2,3}=9.4 \mathrm{~Hz}, 1 \mathrm{H}, \mathrm{H}-2\right) \mathrm{ppm} ;{ }^{13} \mathrm{C}$
NMR: $\delta=163.14(\mathrm{C}=\mathrm{O}), 163.08(\mathrm{C}=\mathrm{O}), 159.16,158.93$ $(2 \times \mathrm{C}-\mathrm{Ar}), \quad 148.63,148.54$ (2× C-pyridine $), 139.25$, $139.13(2 \times \mathrm{C}-\mathrm{Ar}), 139.13$ (C-pyridine), 129.96, 129.92 $(2 \times \mathrm{C}-\mathrm{Ar}), \quad 125.17,125.14$ (2× C-pyridine $), 121.11$, $120.84,114.41,114.17,113.74,112.95(6 \times \mathrm{C}-\mathrm{Ar})$, $104.07\left(\mathrm{C}-2^{\prime}\right), 89.93(\mathrm{C}-1), 84.93\left(\mathrm{C}-3^{\prime}\right), 83.61\left(\mathrm{C}-4^{\prime}\right)$, 83.23 (C-3), 81.51 (C-2), 79.45 (C-4), 78.37 (C-5'), 74.19 $\left(\mathrm{C}-1^{\prime}\right), 69.70(\mathrm{C}-5), 69.23\left(\mathrm{C}-6^{\prime}\right), 66.77$ (C-6), 60.69, 60.47, $59.34,58.53,58.41,58.22\left(6 \times \mathrm{OCH}_{3}\right), 43.66,43.63$ $\left(2 \times \mathrm{CH}_{2} \mathrm{~N}\right)$ ppm; HRMS (ESI): calcd for $\mathrm{C}_{39} \mathrm{H}_{49} \mathrm{~N}_{3} \mathrm{O}_{13} \mathrm{Na}$ $[\mathrm{M}+\mathrm{Na}]^{+} 790.3158$, found 790.3125 .

6,6'-Di-O-[[benzene-1,3-diyl-bis(carbonylaminomethyl)]4,4'-diphenyl]-1',2,3,3',4,4'-hexa-O-methylsucrose

(10c, $\mathrm{C}_{40} \mathrm{H}_{50} \mathrm{~N}_{2} \mathrm{O}_{13}$ )

White solid; yield: $93 \mathrm{mg}(71 \%)$; m.p.: $144{ }^{\circ} \mathrm{C}$; TLC: $R_{f}=0.35 \quad($ AcOEt $) ; \quad[\alpha]_{\mathrm{D}}^{24}=+58.2^{\circ} \mathrm{cm}^{2} \mathrm{~g}^{-1} ; \quad$ IR: $\bar{v}=3,301,3,064,2,982,2,931,2,831,1,649,1,613$, $1,586,1,542,1,514,1,455,1,422,1,359,1,319,1,300$, 1,248, 1,160, 1,101, 1,024, 983, 951, 824, 754, 700, 603, $580 \mathrm{~cm}^{-1} ;{ }^{1} \mathrm{H}$ NMR: $\delta=7.88(\mathrm{~d}, J=7.8 \mathrm{~Hz}, 1 \mathrm{H}$, isophthalic), 7.85 (d, $J=7.6 \mathrm{~Hz}, 1 \mathrm{H}$, isophthalic), 7.56 (s, 1H, isophthalic), $7.45(\mathrm{dd}, J=7.8 \mathrm{~Hz}, 7.6 \mathrm{~Hz}, 1 \mathrm{H}$, isophthalic), $7.22(\mathrm{~d}, J=8.5 \mathrm{~Hz}, 2 \mathrm{H}, \mathrm{Ar}), 7.07$ (d, $J=8.5 \mathrm{~Hz}, 2 \mathrm{H}, \mathrm{Ar}), 6.91(\mathrm{~d}, J=8.5 \mathrm{~Hz}, 2 \mathrm{H}, \mathrm{Ar}), 6.78$ (d, $J=8.5 \mathrm{~Hz}, 2 \mathrm{H}, \mathrm{Ar}$ ), 6.61 (br s, $1 \mathrm{H}, \mathrm{NH}$ ), 6.56 (br s, $1 \mathrm{H}, \mathrm{NH}), 5.55\left(\mathrm{~d}, J_{1,2}=3.7 \mathrm{~Hz}, 1 \mathrm{H}, \mathrm{H}-1\right), 4.45$ (dd, $\left.J=13.9 \mathrm{~Hz}, 5.2 \mathrm{~Hz}, 1 \mathrm{H}, \mathrm{CH}_{2} \mathrm{~N}\right), 4.39(\mathrm{dd}, J=13.8 \mathrm{~Hz}$, $\left.6.7 \mathrm{~Hz}, 1 \mathrm{H}, \mathrm{CH}_{2} \mathrm{~N}\right), 4.35-4.39\left(\mathrm{~m}, 2 \mathrm{H}, \mathrm{H}-6^{\prime}, \mathrm{CH}_{2} \mathrm{~N}\right), 4.32$ $\left(\mathrm{dd}, J=13.8 \mathrm{~Hz}, 4.8 \mathrm{~Hz}, 1 \mathrm{H}, \mathrm{CH}_{2} \mathrm{~N}\right), 4.28(\mathrm{~m}, 1 \mathrm{H}, \mathrm{H}-5)$, $4.20\left(\mathrm{ddd}, J_{5^{\prime}, 4^{\prime}}=8.3 \mathrm{~Hz}, J_{5^{\prime}, 6^{\prime}}=6.7 \mathrm{~Hz}, J_{5^{\prime}, 6^{\prime}}=3.3 \mathrm{~Hz}\right.$, $\left.1 \mathrm{H}, \mathrm{H}-5^{\prime}\right), 4.14\left(\mathrm{~d}, J_{3^{\prime}, 4^{\prime}}=7.9 \mathrm{~Hz}, 1 \mathrm{H}, \mathrm{H}-3^{\prime}\right), 4.13(\mathrm{~m}, 1 \mathrm{H}$, H-6), 4.09 (dd, $\left.J_{6,6}=9.8 \mathrm{~Hz}, J_{6,5}=5.3 \mathrm{~Hz}, 1 \mathrm{H}, \mathrm{H}-6\right)$, $4.05\left(\mathrm{dd}, J_{6^{\prime}, 6^{\prime}}=9.9 \mathrm{~Hz}, J_{6^{\prime}, 5^{\prime}}=3.3 \mathrm{~Hz}, 1 \mathrm{H}, \mathrm{H}-6^{\prime}\right), 4.03$ $\left(\mathrm{dd}, J_{4^{\prime}, 3^{\prime}}=7.9 \mathrm{~Hz}, J_{4^{\prime}, 5^{\prime}}=8.3 \mathrm{~Hz}, 1 \mathrm{H}, \mathrm{H}-4^{\prime}\right), 3.65(\mathrm{~s}, 3 \mathrm{H}$, $\left.\mathrm{CH}_{3}\right), 3.57\left(\mathrm{~d}, J_{1^{\prime}, 1^{\prime}}=11.0 \mathrm{~Hz}, 1 \mathrm{H}, \mathrm{H}-1^{\prime}\right), 3.56(\mathrm{~s}, 3 \mathrm{H}$, $\left.\mathrm{CH}_{3}\right), 3.56(\mathrm{~m}, 1 \mathrm{H}, \mathrm{H}-3), 3.541\left(\mathrm{~s}, 3 \mathrm{H}, \mathrm{CH}_{3}\right), 3.535$ (s, 3H, $\left.\mathrm{CH}_{3}\right), 3.48\left(\mathrm{~s}, 3 \mathrm{H}, \mathrm{CH}_{3}\right), 3.44\left(\mathrm{~s}, 3 \mathrm{H}, \mathrm{CH}_{3}\right), 3.42$ (d, $\left.J_{1^{\prime}, 1^{\prime}}=11.0 \mathrm{~Hz}, 1 \mathrm{H}, \quad \mathrm{H}-1^{\prime}\right), 3.26\left(\mathrm{dd}, J_{4,3}=9.2 \mathrm{~Hz}\right.$, $\left.J_{4,5}=9.8 \mathrm{~Hz}, \quad 1 \mathrm{H}, \quad \mathrm{H}-4\right), \quad 3.18 \quad\left(\mathrm{dd}, \quad J_{2,1}=3.7 \mathrm{~Hz}\right.$, $\left.J_{2,3}=9.6 \mathrm{~Hz}, 1 \mathrm{H}, \mathrm{H}-2\right) \mathrm{ppm} ;{ }^{13} \mathrm{C}$ NMR: $\delta=167.25$ $(\mathrm{C}=\mathrm{O}), 166.87(\mathrm{C}=\mathrm{O}), 158.47,158.42(2 \times \mathrm{C}-\mathrm{Ar}), 134.88$, 134.88, 131.03, 130.99 (4 × C-isophthalic), 130.48, $129.80(2 \times \mathrm{C}-\mathrm{Ar}), 129.71$ (C-isophthalic), 129.71 (2CAr), 128.59 (2C-Ar), 123.88 (C-isophthalic), 114.80 (2CAr), 114.71 (2C-Ar), 103.74 (C-2'), 88.89 (C-1), 84.64 (C$\left.3^{\prime}\right), 83.72\left(\mathrm{C}-4^{\prime}\right), 83.28$ (C-3), 81.68 (C-2), 79.84 (C-4), $78.89\left(\mathrm{C}-5^{\prime}\right), 74.22\left(\mathrm{C}-1^{\prime}\right), 69.77(\mathrm{C}-5), 69.44\left(\mathrm{C}-6^{\prime}\right), 67.69$

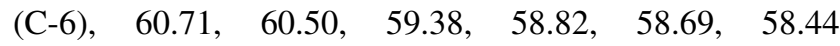
$\left(6 \times \mathrm{OCH}_{3}\right), \quad 43.72,43.39\left(2 \times \mathrm{CH}_{2} \mathrm{~N}\right) \quad$ ppm; HRMS (ESI): calcd for $\mathrm{C}_{40} \mathrm{H}_{50} \mathrm{~N}_{2} \mathrm{O}_{13} \mathrm{Na}[\mathrm{M}+\mathrm{Na}]^{+}$789.3202, found 789.3203 . 
6,6'-Di-O-[[pyridine-1,3-diyl-bis(carbonylaminomethyl)]4,4'-diphenyl]-1',2,3,3',4,4'-hexa-O-methylsucrose

(11c, $\mathrm{C}_{39} \mathrm{H}_{49} \mathrm{~N}_{3} \mathrm{O}_{13}$ )

White solid; yield: $97 \mathrm{mg}(74 \%)$; m.p.: $115^{\circ} \mathrm{C}$; TLC: $R_{f}=0.37 \quad(\mathrm{AcOEt}) ; \quad[\alpha]_{\mathrm{D}}^{21}=+28.4^{\circ} \mathrm{cm}^{2} \mathrm{~g}^{-1} ; \quad$ IR: $\bar{v}=3,330,2,982,2,932 ， 2,831,1,671,1,613,1,585$, $1,535,1,514,1,449,1,363,1,301,1,287,1,248,1,151$, 1,100, 1,023, 1,003, 984, 949, 879, 827, 753, 677, 646, 603, $582 \mathrm{~cm}^{-1}$; ${ }^{1} \mathrm{H}$ NMR: $\delta=8.35(\mathrm{dd}, J=7.8 \mathrm{~Hz}, 1.2 \mathrm{~Hz}$, $1 \mathrm{H}$, pyridine), 8.32 (dd, $J=7.8 \mathrm{~Hz}, 1.2 \mathrm{~Hz}, 1 \mathrm{H}$, pyridine), $8.05(\mathrm{t}, J=7.8 \mathrm{~Hz}, 1 \mathrm{H}$, pyridine), $7.69(\mathrm{dd}, J=4.6 \mathrm{~Hz}$, $5.4 \mathrm{~Hz}, 1 \mathrm{H}, \mathrm{NH}), 7.63$ (dd, $J=4.6 \mathrm{~Hz}, 4.8 \mathrm{~Hz}, 1 \mathrm{H}, \mathrm{NH}$ ), $7.26(\mathrm{~d}, J=8.7 \mathrm{~Hz}, 2 \mathrm{H}, \mathrm{Ar}), 7.02(\mathrm{~d}, J=8.7 \mathrm{~Hz}, 2 \mathrm{H}, \mathrm{Ar})$, $6.93(\mathrm{~d}, J=8.7 \mathrm{~Hz}, 2 \mathrm{H}, \mathrm{Ar}), 6.73(\mathrm{~d}, J=8.7 \mathrm{~Hz}, 2 \mathrm{H}, \mathrm{Ar})$, $5.52\left(\mathrm{~d}, J_{1,2}=3.7 \mathrm{~Hz}, 1 \mathrm{H}, \mathrm{H}-1\right), 4.67(\mathrm{dd}, J=5.5 \mathrm{~Hz}$, $\left.14.2 \mathrm{~Hz}, 1 \mathrm{H}, \mathrm{CH}_{2} \mathrm{~N}\right), 4.57(\mathrm{dd}, J=5.9 \mathrm{~Hz}, 14.9 \mathrm{~Hz}, 1 \mathrm{H}$, $\left.\mathrm{CH}_{2} \mathrm{~N}\right), 4.53\left(\mathrm{dd}, J_{6^{\prime}, 5^{\prime}}=6.8 \mathrm{~Hz}, J_{6^{\prime}, 6^{\prime}}=10.0 \mathrm{~Hz}, 1 \mathrm{H}\right.$, H-6' $), 4.46\left(\mathrm{dd}, J=4.2 \mathrm{~Hz}, 14.9 \mathrm{~Hz}, 1 \mathrm{H}, \mathrm{CH}_{2} \mathrm{~N}\right), 4.42$ (dd, $J=4.1 \mathrm{~Hz}, \quad 14.2 \mathrm{~Hz}, \quad 1 \mathrm{H}, \quad \mathrm{CH}_{2} \mathrm{~N}$ ), 4.34 (ddd, $\left.J_{5,6}=1.3 \mathrm{~Hz}, J_{5,6}=6.6 \mathrm{~Hz}, J_{5,4}=10.3 \mathrm{~Hz}, 1 \mathrm{H}, \mathrm{H}-5\right)$, $4.24\left(\mathrm{ddd}, J_{5^{\prime}, 6^{\prime}}=3.2 \mathrm{~Hz}, J_{5^{\prime}, 6^{\prime}}=6.8 \mathrm{~Hz}, J_{5^{\prime}, 4^{\prime}}=7.6 \mathrm{~Hz}\right.$, $\left.1 \mathrm{H}, \mathrm{H}-5^{\prime}\right), 4.22\left(\mathrm{dd}, J_{6,5}=1.3 \mathrm{~Hz}, J_{6,6}=9.6 \mathrm{~Hz}, 1 \mathrm{H}\right.$, $\mathrm{H}-6), 4.15\left(\mathrm{dd}, J_{4^{\prime}, 3^{\prime}}=7.9 \mathrm{~Hz}, J_{4^{\prime}, 5^{\prime}}=7.6 \mathrm{~Hz}, 1 \mathrm{H}, \mathrm{H}-4^{\prime}\right)$, $4.12\left(\mathrm{~d}, J_{3^{\prime}, 4^{\prime}}=7.9 \mathrm{~Hz}, 1 \mathrm{H}, \mathrm{H}-3^{\prime}\right), 4.10\left(\mathrm{dd}, J_{6,5}=6.6 \mathrm{~Hz}\right.$, $\left.J_{6,6}=9.6 \mathrm{~Hz}, \quad 1 \mathrm{H}, \quad \mathrm{H}-6\right), 4.08 \quad\left(\mathrm{dd}, J_{6^{\prime}, 6^{\prime}}=10.0 \mathrm{~Hz}\right.$, $\left.J_{5^{\prime}, 6^{\prime}}=3.2 \mathrm{~Hz}, 1 \mathrm{H}, \mathrm{H}-6^{\prime}\right), 3.65\left(\mathrm{~s}, 3 \mathrm{H}, \mathrm{CH}_{3}\right), 3.58(\mathrm{~s}, 6 \mathrm{H}$, $\left.\mathrm{CH}_{3}\right), 3.57\left(\mathrm{~d}, J_{1^{\prime}, 1^{\prime}}=11.0 \mathrm{~Hz}, 1 \mathrm{H}, \mathrm{H}-1^{\prime}\right), 3.55(\mathrm{~m}, 4 \mathrm{H}$, $\left.\mathrm{H}-3, \mathrm{CH}_{3}\right), 3.47$ (s, 3H, $\left.\mathrm{CH}_{3}\right), 3.45$ (s, 3H, $\left.\mathrm{CH}_{3}\right), 3.40$ (d, $\left.J_{1^{\prime}, 1^{\prime}}=11.0 \mathrm{~Hz}, 1 \mathrm{H}, \mathrm{H}-1^{\prime}\right), 3.15\left(\mathrm{dd}, J_{2,1}=3.7 \mathrm{~Hz}\right.$, $\left.J_{2,3}=9.6 \mathrm{~Hz}, \quad 1 \mathrm{H}, \quad \mathrm{H}-2\right), \quad 3.13 \quad\left(\mathrm{dd}, \quad J_{4,3}=8.7 \mathrm{~Hz}\right.$, $\left.J_{4,5}=10.3 \mathrm{~Hz}, 1 \mathrm{H}, \mathrm{H}-4\right) \mathrm{ppm} ;{ }^{13} \mathrm{C} \mathrm{NMR}: \delta=162.90$ $(\mathrm{C}=\mathrm{O}), 162.77(\mathrm{C}=\mathrm{O}), 158.49,158.42(2 \times \mathrm{C}-\mathrm{Ar}), 148.59$, 148.43, 139.23 (3 × C-pyridine), 129.70 (2C-Ar), 129.61, $129.11(2 \times \mathrm{C}-\mathrm{Ar}), \quad 128.17$ (2C-Ar), 124.81, 124.76 (2 $\times$ C-pyridine), $114.92 \quad$ (2C-Ar), $114.70 \quad$ (2C-Ar), $103.71\left(\mathrm{C}-2^{\prime}\right), 88.70$ (C-1), $84.51\left(\mathrm{C}-4^{\prime}\right), 83.70\left(\mathrm{C}-3^{\prime}\right)$, 83.34 (C-3), 81.73 (C-2), 80.20 (C-4), 79.01 (C-5'), 73.99 $\left(\mathrm{C}-1^{\prime}\right), 69.93$ (C-5), $69.42\left(\mathrm{C}-6^{\prime}\right), 68.06$ (C-6), 60.68, 60.47, 59.34, 58.91, 58.75, $58.42\left(6 \times \mathrm{OCH}_{3}\right), 43.66,43.06$ $\left(2 \times \mathrm{CH}_{2} \mathrm{~N}\right)$ ppm; HRMS (ESI): calcd for $\mathrm{C}_{39} \mathrm{H}_{49} \mathrm{~N}_{3} \mathrm{O}_{13} \mathrm{Na}$ $[\mathrm{M}+\mathrm{Na}]^{+}$790.3158, found 790.3196 .

Acknowledgments The support from grant POIG.01.01.02-14-102/ 09 (part-financed by the European Union within the European Regional Development Fund) is acknowledged.
Open Access This article is distributed under the terms of the Creative Commons Attribution License which permits any use, distribution, and reproduction in any medium, provided the original author(s) and the source are credited.

\section{References}

1. Steed JW, Atwood JL (2009) Supramolecular chemistry, 2nd edn. Wiley, Chichester

2. Bakó P, Keglevich G, Rapi Z, Tőke L (2012) Curr Org Chem $16: 297$

3. Bakó P, Makó A, Keglevich G, Kubinyi M, Pál K (2005) Tetrahedron Asymmetry 16:1861

4. Makó A, Szöllősy Á, Keglevich G, Menyhárd DK, Bakó P, Tőke L (2008) Monatsh Chem 139:525

5. Rapi Z, Szabó T, Keglevich G, Szöllősy Á, Drahos L, Bakó P (2011) Tetrahedron Asymmetry 22:1189

6. Bakó T, Bakó P, Keglevich G, Báthori N, Czugler M, Tatai J, Novák T, Parlagh G, Tőke L (2003) Tetrahedron Asymmetry 14:1917

7. Bakó P, Rapi Z, Keglevich G, Szabó T, Sóti PL, Vígh T, Grün A, Holczbauer T (2011) Tetrahedron Lett 52:1473

8. Rapi Z, Bakó P, Keglevich G, Szöllősy Á, Drahos L, Botyánszki A, Holczbauer T (2012) Tetrahedron Asymmetry 23:489

9. Xie J, Ménand M, Maisonneuve S, Métivier R (2007) J Org Chem 72:5980

10. Hsieh YC, Chir JL, Wu HH, Guo CQ, Wu AT (2010) Tetrahedron Lett 51:109

11. Yang ST, Liao DJ, Chen SJ, Hu CH, Wu AT (2012) Analyst 137:1553

12. Jarosz S, Listkowski A (2003) J Carbohydr Chem 22:753

13. Jarosz S, Listkowski A (2006) Can J Chem 84:492

14. Lewandowski B, Jarosz S (2010) Org Lett 12:2532

15. Lewandowski B, Jarosz S (2008) Chem Commun 47:6399

16. Sansone F, Baldini L, Casnati A, Lazzarotto M, Ugozzoli F, Ungaro R (2002) Proc Natl Acad Sci USA 99:4842

17. Kima SK, Sessler JL (2010) Chem Soc Rev 39:3784

18. Santos SM, Costa PJ, Lankshear MD, Beer PD, Félix V (2010) J Phys Chem B 114:11173

19. Gasparrini F, Misiti D, Pierini M, Villani C (2002) Org Lett 4:3993

20. Evans NH, Rahman H, Leontiev AV, Greenham ND, Orlowski GA, Zeng Q, Jacobs RMJ, Serpell CJ, Kilah NL, Davis JJ, Beer PD (2012) Chem Sci 3:1080

21. Evans NH, Serpell CJ, Beer PD (2011) Chem Commun 47:8775

22. Lang T, Graf E, Kyritsakas N, Hosseini MW (2012) Chem Eur J 18:10419

23. Potopnyk MA, Cmoch P, Jarosz S (2012) Org Lett 14:4258 